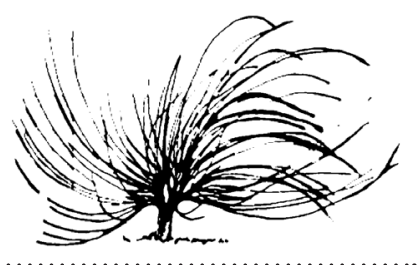

\title{
La ineludible visión andragógica para la Universidad Nacional de Costa Rica (UNA)
}

\author{
German Eduardo González-Sandoval ${ }^{1}$ \\ Universidad Nacional \\ Costa Rica \\ germangs24@gmail.com
}

"La Andragogía es el arte y ciencia de ayudar a aprender a los adultos, basándose en suposiciones acerca de las diferencias entre niños y adultos"

Malcolm Knowles

\begin{abstract}
Resumen
Este ensayo versa sobre la necesidad de implementar los principios de la andragogía en la Universidad Nacional. Por ende, se hace un repaso general por su normativa, la cual respalda la eventual inclusión de la andragogía en el nivel institucional; se presenta un esbozo comparativo sobre sus alcances con los de la pedagogía, sus características generales, sus beneficios para el aprendizaje de los adultos y sus principios básicos. El texto tiene la finalidad de generar una reflexión para desarrollar acciones y lineamientos en los que se enmarque el quehacer académico de la Universidad, tomando en cuenta las características para el aprendizaje de la persona
\end{abstract}

\section{(c) (1) $\odot \Theta$}

Recibido: 17 de julio de 2017. Aprobado: 24 de setiembre de 2018.

http://dx.doi.org/10.15359/rep.13-2.2

1. Licenciado en Orientación Educativa y Administración Educativa de la Universidad Nacional de Costa Rica y Magíster en Educación con énfasis en Docencia Universitaria de la Universidad Nacional de Costa Rica. Desde el 2007 se ha desempeñado como docente, investigador, extensionista y, actualmente, como director de la División de Educación para el Trabajo del Centro de Investigación y Docencia en Educación de la Universidad Nacional. 
adulta. Se desarrolla un modelo con cuatro elementos básicos por contemplar en todo modelo institucional, a saber: el concepto general del aprendizaje, el papel de la experiencia del aprendiz, disposición para aprender y orientación al aprendizaje. Para ahondar en el análisis, se propone un concepto holístico sobre el ser humano desde las dimensiones biológica, social, psicológica y legal, su conceptualización específica señalando las dimensiones cognitiva, física, emocional y personal. Finalmente, se plantean algunas conclusiones que confirman la necesidad de implementar la andragogía en la enseñanza superior y en el quehacer de la Universidad Nacional, para garantizar el abordaje en los procesos educativos de los estudiantes universitarios que son del grupo etario de personas adultas.

Palabras claves: andragogía, Universidad Nacional, pedagogía, enseñanza superior.

\begin{abstract}
This essay deals with the need to implement the principles of andragogy at Universidad Nacional in Costa Rica. Therefore, a general review is made about its regulations which supports the eventual inclusion of andragogy at the institutional level, and a comparative outline is presented on its scope with those of pedagogy, its general characteristics, its benefits for learning for adults and their basic principles. The purpose of the essay is to generate a reflection to develop actions and guidelines in which the academic work of the university is framed taking into account the characteristics of adult learning. A model is developed with four basic elements to be considered in any institutional model to know the general concept of learning, the role of the learner's experience, willingness to learn and guidance to learn. To delve into the analysis, a holistic concept about the human being is proposed from the biological, social, psychological and legal dimensions, and its specific conceptualization pointing out the cognitive, physical, emotional, and personal dimensions. Finally, some conclusions are presented that confirm the need to implement andragogy in higher
\end{abstract}


education and in the work of Universidad Nacional to ensure the approach in the educational processes of university students who are adults.

Keywords: andragogy, Universidad Nacional, pedagogy, higher education.

\section{Introducción}

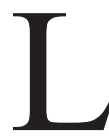

a pretensión de este ensayo es generar espacios de reflexión y acción para promover diálogos en la comunidad universitaria, con el fin de concretar la necesidad de determinar el abordaje de la población de estudiantes adultos de la Universidad Nacional (UNA), más allá de convertirse en un documento para profundizar en los alcances conceptuales y prácticos de la andragogía como parte del modelo institucional. Aun así, se problematiza el requerimiento de integrarla al poner en marcha un modelo congruente con la visión, la misión y los principios, especialmente atendiendo el enfoque humanista implícito desde la fundación como universidad.

Es importante reconocer la iniciativa institucional por elaborar un modelo pedagógico en el cual se expresan los principios y lineamientos en los que se enmarca el quehacer académico de la Universidad Nacional (UNA). Sin embargo, es necesario identificar que esta iniciativa, aunque política y sociocultural, debe ser fundamentalmente una tarea académica y llaman la atención, en el planteamiento de este modelo, aspectos como la necesidad de alcanzar el objetivo de promover la identidad y el sentido de pertenencia institucional; fortalecer los procesos de enseñanza y aprendizaje; mejorar la gestión curricular, la evaluación y la oferta académica; formar profesionales competentes, con una visión humanista, y promover el desarrollo profesional del personal académico.

Se observa como un gran reto alcanzar el cumplimiento de las propuestas anteriores y se ha señalado como alternativa darlas a conocer a la comunidad universitaria y generar un compromiso con dicho modelo; no obstante, más allá de la necesidad de darle divulgación, mediante programas de la Vicerrectoría de Docencia, se ha determinado como estrategia la ruta por seguir en el quehacer de nuestra universidad. Es menester, entonces, crear espacios para favorecer su discusión y 
análisis crítico con "toda" la comunidad universitaria y generar la apropiación tanto consiente como voluntaria de los principios y fundamentos coherentes con la fundación de la UNA y el recorrido institucional por ya cuarenta y cinco años, favoreciendo el respeto a la diversidad representada en el total de la población estudiantil universitaria.

De igual manera, sobresale de dicho documento una tendencia generalizada y poco detallada en la cual se hace necesario definir los aspectos de la práctica educativa que se pretende, no se visualiza en el modelo pedagógico de la UNA alguna descripción o explicación específica del acto o práctica educativa, ni se describen las características del sujeto de la misma, en el quehacer de la docencia universitaria, lo que se considera necesario pues los saberes disciplinarios no garantizan el conocimiento de las bases y principios del saber pedagógico y es precisamente un modelo pedagógico institucional el encargado de facilitar y promover ese quehacer académico en la educación superior.

Se debe proyectar y divulgar a la comunidad nacional e internacional el modelo de enseñanza al cual se adscribe la Universidad Nacional, en el que se refleja toda la acción medular de la academia, sin distinción en áreas de formación, donde los protagonistas del acto educativo posean claridad absoluta de la concepción en su ejecución. Aquí sugiero también valorar la inclusión de los principios andragógicos que no se integran en dicho modelo. Considero que para lograrlo se deben establecer espacios de análisis y discusión, o bien convocar a un congreso universitario, con el afán de trascender las discusiones políticas y administrativas características de estos espacios, para dar el paso académico que la sociedad actual demanda, aunque partiendo de la experiencia académica atesorada en estos años, escuchando las voces de sus protagonistas.

A pesar de contar con el nuevo Estatuto Orgánico puesto en marcha desde el pasado 17 de agosto de 2015, como resultado de un extenso congreso universitario lleno de intensas jornadas dialógicas, la temática acá propuesta no fue uno de los temas tratados. Esto último corresponde a la atención y al abordaje de una cantidad importante de estudiantes ubicados en rangos de edades propios de las etapas adultas que ingresan a la universidad con la misma ilusión de alcanzar sus metas de profesionalización, ya sea por primera vez, por reingreso para finalizar algún proceso incompleto, rezago, o bien por alcanzar una especialización de su perfil profesional. Personalmente, señalo la invisibilización de esta 
población estudiantil con rasgos específicos, pues en el discurso colectivo se hace referencia a una comunidad estudiantil adolescente y joven con características propias a las de nuevo ingreso, que continúa sus estudios en una línea de lo asumido "como normal", o sea al finalizar los estudios secundarios ingresan a la educación superior.

Por todo lo anterior, se hace necesario profundizar en el tema de la andragogía, pues, tal y como lo plantean Knowles, Holton y Swanson (2005), esta es un conjunto de principios fundamentales sobre el aprendizaje de los adultos que se aplica a todas las situaciones de tal aprendizaje, las cuales permiten diseñar y guiar procesos docentes más eficaces. Esta información sugiere la pregunta: ¿la andragogía es contraria o antagónica a la pedagogía? Aunque algunos críticos han señalado diferencias, ciertas de ellas se presentan con una finalidad práctica más allá de generar un debate sin sentido, alejado del objetivo educativo de encontrar las mejores estrategias para lograr hacer efectivo el proceso de enseñanza y aprendizaje, así como para generar acciones de análisis y reflexión con las que el estudiante, de manera crítica, sea el constructor de su propio destino para su desarrollo permanente.

Es importante destacar la principal deferencia que se le asigna históricamente a la pedagogía como una ciencia relacionada con la enseñanza de poblaciones infantiles y juveniles, enfocada principalmente en el contenido, según Knowles, Holton y Swanson (2005), en los modelos pedagógicos:

se le asigna al profesor la responsabilidad por las decisiones sobre lo que hay que aprender, cómo se aprenderá y si se ha conseguido aprender. Es una educación dirigida por el profesor, dejándole al alumno el sumiso papel de atender las instrucciones de aquel. (p. 66)

Las diferencias que sobresalen entre la pedagogía y la andragogía, se pueden visualizar desde el planteamiento de Lloyd (2015), quien las presenta en cuatro elementos básicos que deben estar plasmados en todo modelo institucional, a saber: primero el aprendiz, su concepto general de estudiante o, de manera más integral, el ser humano aprendiente; segundo la experiencia del aprendiz o su bagaje experiencial; tercero la disponibilidad para aprender o su actitud ante los procesos de aprendizaje, y cuarto su orientación al aprendizaje. Cada uno de los elementos establece diferencias sustantivas, como bien se puede observar en la tabla 1 : 
Tabla 1.

\section{Diferencias entre pedagogía y andragogía}

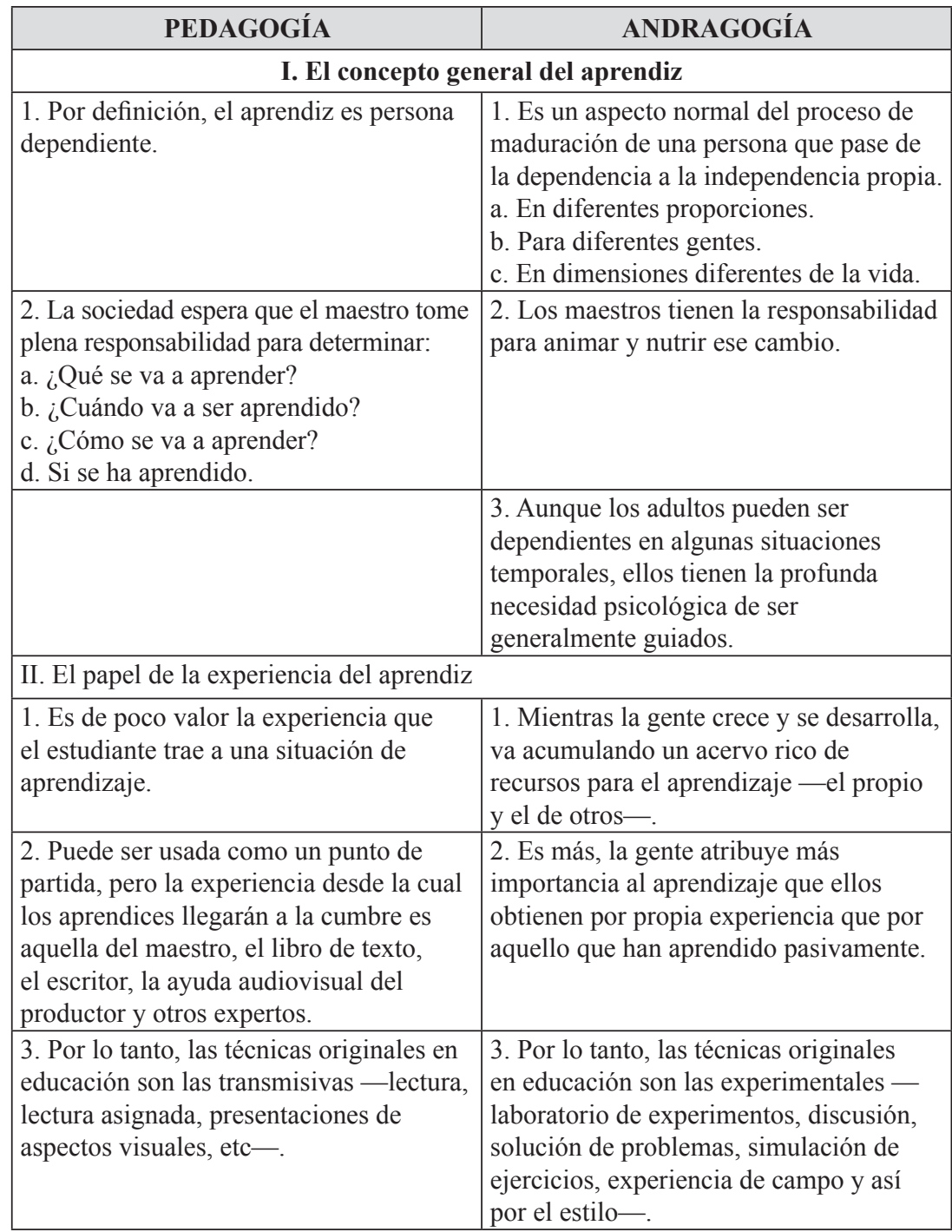




\begin{tabular}{|c|c|}
\hline PEDAGOGÍA & ANDRAGOGÍA \\
\hline \multicolumn{2}{|c|}{ III. ¡Listos para aprender! } \\
\hline $\begin{array}{l}\text { 1. La gente está lista para aprender todo } \\
\text { lo que la sociedad (especialmente la } \\
\text { escuela) dice que debe aprender. }\end{array}$ & $\begin{array}{l}\text { 1. Las personas se interesan en aprender } \\
\text { algo cuando ellas mismas experimentan } \\
\text { la necesidad de aprenderlo, para } \\
\text { enfrentarse más satisfactoriamente con } \\
\text { las tareas o problemas de la vida real. }\end{array}$ \\
\hline $\begin{array}{l}\text { 2. Esta ejerce presiones bastante fuertes } \\
\text { sobre los educandos — como el miedo y } \\
\text { el fracaso-. }\end{array}$ & $\begin{array}{l}\text { 2. El educador tiene la responsabilidad de } \\
\text { crear condiciones y proveer herramientas } \\
\text { y procedimientos para ayudar a sus } \\
\text { aprendices a descubrir sus propias } \\
\text { necesidades. }\end{array}$ \\
\hline $\begin{array}{l}\text { 3. La mayoría de las personas de la } \\
\text { misma edad se preparan para aprender } \\
\text { cosas iguales. }\end{array}$ & $\begin{array}{l}\text { 3. Los programas de aprendizaje deben } \\
\text { ser organizados por categorías como la } \\
\text { vida/aplicación y seguir una secuencia, } \\
\text { de acuerdo con la buena disposición de } \\
\text { los estudiantes para aprender. }\end{array}$ \\
\hline $\begin{array}{l}\text { 4. Por lo tanto, el aprendizaje debe ser } \\
\text { organizado en un limpio y claro currículo } \\
\text { para todos los aprendices. Este se } \\
\text { controla con una constante progresión. }\end{array}$ & \\
\hline \multicolumn{2}{|c|}{ IV. Orientación al aprendizaje } \\
\hline $\begin{array}{l}\text { 1. Los estudiantes ven la educación como } \\
\text { proceso de adquisición de contenido en } \\
\text { el sentido de tema/materia (cursos). }\end{array}$ & $\begin{array}{l}\text { 1. Los aprendices ven la educación como } \\
\text { un proceso de desarrollo de crecida } \\
\text { competencia, para llevar a cabo su pleno } \\
\text { potencial en la vida. }\end{array}$ \\
\hline $\begin{array}{l}\text { 2. Los educandos entienden que la } \\
\text { mayoría de esos tema/materias no será } \\
\text { útil hasta más tarde en su vida. }\end{array}$ & $\begin{array}{l}\text { 2. Ellos quieren poder aplicar de } \\
\text { inmediato cualquier conocimiento y } \\
\text { habilidad que hayan obtenido. Desean } \\
\text { vivir el mañana con mayor satisfacción. }\end{array}$ \\
\hline $\begin{array}{l}\text { 3. En consecuencia, el currículo debe } \\
\text { ser organizado en unidades de cursos } \\
\text { que siguen la lógica del tema (desde la } \\
\text { historia prehistórica hasta la moderna, de } \\
\text { lo simple a lo complejo, en materias de } \\
\text { matemáticas y ciencia). }\end{array}$ & $\begin{array}{l}\text { 3. En consecuencia, las experiencias del } \\
\text { aprendizaje deben ser organizadas en las } \\
\text { categorías de competencia y desarrollo. }\end{array}$ \\
\hline $\begin{array}{l}\text { 4. El aprendizaje se orienta alrededor } \\
\text { de temas preestablecidos que serán de } \\
\text { utilidad en años posteriores. }\end{array}$ & $\begin{array}{l}\text { 4. El aprendizaje se orienta alrededor de } \\
\text { aquello que sea útil para ahora mismo } \\
\text { experimentar la vida real. }\end{array}$ \\
\hline
\end{tabular}

Nota: Tabla elaborada con base en información tomada de Lloyd (2015). 
La tabla 1, además de presentar claramente las diferencias entre una y otra disciplina desde los cuatro elementos sugeridos, nos plantea los componentes necesarios para identificar - me atrevo a decir-, a modo de lista de cotejo, cuál es la manera o metodología con que se desarrollan los procesos de mediación. Se indica, por ejemplo, la necesidad de promover una educación con sentido crítico y apegada a las necesidades de formación del estudiante, con especial énfasis en el aprender haciendo. Son estos aspectos los que deben ser tomados en cuenta en el nivel institucional, con la finalidad de promover espacios de mediación oportunos y adecuados para la atención y el abordaje de la población adulta en todos los procesos de formación universitaria, desde lo curricular hasta lo extracurricular.

Con el propósito de comprender la forma en que la andragogía posibilita y favorece el desarrollo de los procesos educativos, se pueden valorar las cuatro fases sugeridas por Pérez (2009), en los fundamentos de su "modelo andragógico", claramente definidas así:

- Una primera fase de su finalidad es mantener, consolidar y enriquecer los intereses del adulto, que le permitan abrir nuevas perspectivas de vida.

- A fin de mostrarle nuevos rumbos prospectivos y promover el principio de que, en todo grupo humano, educarse es progresar, la segunda fase es de orientación.

- La tercera fase consiste en actualizar al adulto respecto a los conocimientos, valores y habilidades intelectuales que le permitan generar procesos de auto aprendizaje.

- Interpretar los factores y variables de nuestra historicidad como seres humanos; es la cuarta fase. Y debería de permitir el reconocer, valorar y articular las viejas y nuevas experiencias en la configuración de un proyecto de vida personal y social. (p. 31)

Por lo anterior, es necesario comprender que la educación superior debe favorecer una mediación intencionada al desarrollo de un proyecto personal, que se cimentará en el conocimiento de nuevas formas de ver la vida y nuevas expectativas que le permitan al estudiante la actualización para el desenvolvimiento de sus procesos de autoaprendizaje, tomando en cuenta los aspectos de nuestra propia historia de vida. 
Entre las características actuales de la UNA como institución, es importante señalar la gran cantidad de oportunidades y ventajas que se ofrecen a la comunidad estudiantil y, en general, mediante diferentes estrategias como el sistema de becas, los programas de promoción estudiantil, la extensión e investigación y su reconocido respeto a la diversidad en todas sus dimensiones, lo cual genera la existencia de una variada gama de estudiantes: los de primer ingreso, algunos con sus apenas recién cumplidos dieciocho años y otros más que no son recién graduados de la secundaria, o bien el grupo de estudiantes ya personas adultas o adultas jóvenes, quienes, por algún motivo, se vieron obligadas a aplazar o demorar tanto su ingreso como la culminación de sus estudios universitarios, así como los estudiantes que han valorado la opción de continuar estudios de grado y posgrado. Ellos, de la misma manera, pertenecen al grupo etario mencionado anteriormente y se convierten en una población que requiere atención oportuna en igualdad de condiciones para alcanzar el objetivo de la formación universitaria. Por lo anterior, es necesario implementar como casa de estudios superiores una cultura de derechos humanos que favorezca una ampliación de la cobertura y la oferta de la educación superior en general, tanto en el nivel de grado como en el de posgrados, con metodologías integradoras e incluyentes en una academia que asuma la docencia como un acto de respeto y compromiso, al igual que brinde oportunidades para todos.

La reflexión presentada permite iniciar con el planteamiento central de este ensayo, el cual considera necesario resaltar que otras instituciones de educación superior han consolidado esfuerzos para ofrecer alternativas competentes y oportunas de formación universitaria a poblaciones de adultos. Entre ellas, aparece la Universidad Estatal a Distancia en Costa Rica y en España, la Universidad Andragógica Autónoma en Estados Unidos, la Universidad Nacional Experimental Simón Rodríguez en Venezuela y otras más que pueden fácilmente ser consultadas mediante su buscador de preferencia en Internet. Con una lectura acerca de su descripción, se evidencia que estas instituciones se han comprometido con una oferta adaptada a las necesidades, realidades y experiencias de esta población.

Aunque se puede pensar que el tema de la educación andragógica es tan complejo que es difícil ponerla en marcha, se hace importante conocer los siete elementos indicados por el Instituto Nacional para la 
Educación de Adultos (INEA) (2007, citado por Alonso, 2012), de la siguiente manera:

1. Establecer un ambiente adecuado: se debe propiciar un ambiente cálido, de diálogo y de respeto mutuo en el cual los participantes interactúen sin temor.

2. Planeamiento de la lección: el facilitador de la sesión debe planificar, concienzudamente, el tema y la metodología por usarse y explicar cuál es el propósito de cada uno de los procedimientos (técnicas) para llegar al descubrimiento del nuevo conocimiento.

3. Diagnóstico de las necesidades de estudio: se debe construir un modelo basado en competencias (conocimientos, habilidades y actitudes) que intervienen en el proceso educativo del aprendiente, con el fin de ayudarlo.

4. Establecer objetivos: consiste en transformar las necesidades detectadas en el elemento anterior, para convertirlas en objetivos significativos y medibles.

5. Elaborar un plan de estudios: es elaborar un programa que contenga objetivos, recursos y estrategias para alcanzar los objetivos.

6. Realizar actividades de estudio: investigación individual, debates, conferencias, diálogos, entrevistas, panel, lecturas, juego de roles, análisis de casos, asesorías, etc.

7. Evaluar los resultados del estudio: se deben desarrollar instrumentos eficientes para evaluar los resultados del proceso andragógico. (p. 20)

Tal y como se infiere claramente de los elementos anteriores, las tareas son congruentes con las acciones académicas que no dudo ya se realizan en el entorno educativo de la UNA, pues son aspectos que se plantean tanto en la normativa como en los lineamientos emanados de los procesos de autoevaluación y acreditación de la calidad.

Entonces, se puede conceptualizar integralmente al adulto como tal, valorando, desde la perspectiva del autor, cinco dimensiones básicas y fundamentales para la comprensión del ser humano, aunque no excluyentes de otras como la física o la vocacional. La primera define sus funciones biológicas para su existencia y su reproducción; la segunda posiciona al adulto en su interrelación social y cultura que le impone ciertos compromisos específicos; una tercera le establece sus 
responsabilidades legales como ser sujeto de derechos y deberes, el rol que asuma como adulto; la cuarta (dimensión psicológica) plantea que desde su autoconcepto como ser humano asume las responsabilidades de su propia vida y rige sus actos; la quinta (dimensión espiritual) procura el desarrollo integral y holístico de todas sus necesidades humanas. Así se visualiza cómo se traslapa cada una de dichas dimensiones, de manera simultánea, en las acciones de su diario vivir como persona y, a la vez, son los elementos que deben sustentar el diseño de los procesos educativos, para que logren una pertinencia con la población descrita y congruente con las características específicas de un grupo de estudiantes al que no se le ha brindado toda su atención, a saber:

\section{Figura 1.}

\section{Dimensiones básicas para la comprensión del ser humano BIOLÓGICA \\ Ser humano con capacidad de reproducción.}

\section{ESPIRITUAL}

Atención de todas las necesidades humanas su atención integral y holística en su preocupación por su desarrollo total.

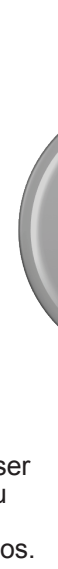

SOCIOCULTURAL

Persona que asume el rol social y cultural, tareas y responsabilidades propias de adulto.

\section{LEGAL}

La persona alcanza la edad para ejercer el derecho al voto, obtiene reconocimiento para asumir compromisos sin el consentimiento de los padres u otro adulto.

Nota: Figura de elaboración propia del autor.

De la figura 1, se desprende una visión holística del ser humano, en la cual se engarzan insoslayablemente cinco dimensiones fundamentales, aunque no son únicas ni definitivas, pues se debe visualizar, en la etapa adulta, la integración de elementos como la vida en pareja o en familia, la conciencia, la vocación, su vinculación laboral, o bien la 
consolidación de un proyecto de vida, entre otros, que con las dinámicas de un mundo globalizado son cada día más demandantes. Entre esos otros elementos es posible mencionar los siguientes:

- La dimensión emocional, integrada tanto por los sentimientos como por las emociones, define la manera en que el individuo se enfrenta en interacción con el mundo circundante.

- La dimensión personal, constituida por el autoconcepto, la autoimagen y la autoestima; cuanto más se conozca el ser humano a sí mismo, en esa medida, reconocerá a los otros y su posición en la sociedad.

- La dimensión física, definida por los procesos de formación y desarrollo corporal, además se integra por la promoción de un estado de bienestar adecuado, que contiene los aspectos propios del área de la sexualidad, pues en la etapa de la adultez se tiene la capacidad del disfrute del cuerpo y del contacto con otras personas y objetos considerados parte integral del propio ser.

- La dimensión cognitiva, determinada por la manera de construir y deconstruir el conocimiento, el desarrollo de competencias, habilidades y destrezas en un marco de compromiso personal y social, es el desenvolvimiento tanto de la inteligencia en general como de la emocional.

Mención aparte, aunque no menos trascendental, es el desarrollo espiritual como una dimensión más, haciendo referencia a los aspectos que se relacionan con experiencias sensoriales propias del descubrimiento del yo interior y de encontrarle sentido a la existencia para vivirla con apego a promover la satisfacción, la felicidad, la plenitud y el empoderamiento como ser integral.

Ahora bien, tomando en cuenta la comprensión del ser adulto desde las dimensiones ya señaladas, es importante retomar el escenario que desde el quehacer universitario se puede establecer como fundamento para atender las necesidades de poblaciones adultas, según lo señalado en el nuevo Estatuto Orgánico (UNA, 2015), en su título I, capítulo único: principios, valores y fines, para su inserción adecuada en la UNA, de la siguiente manera: 


\section{ARTÍCULO 1. PRINCIPIOS}

Los principios que sustentan el quehacer universitario son:

a. Humanismo. La Universidad Nacional promueve la justicia, el bien común, el respeto irrestricto a la dignidad humana y a los derechos de las personas y de la naturaleza.

c. Inclusión. La inclusión de los sectores menos favorecidos por razones económicas, culturales o por discapacidad, se garantiza mediante una oferta académica, políticas de admisión y programas de becas especialmente dirigidos a esos grupos.

f. Conocimiento transformador. Mediante una acción sustantiva innovadora y creativa, la Universidad procura formar personas analíticas, críticas y propositivas que conduzcan al desarrollo de mejores condiciones humanas individuales y sociales. (p. 19)

En cuanto a tales principios, se resalta el humanismo como el medio para poner, a modo de eje central, la acción universitaria al ser humano; la inclusión como una estrategia para favorecer los sectores con menos posibilidades, siendo los adultos uno de ellos, y el conocimiento transformador como elemento promotor de una formación analítica, crítica y propositiva para desarrollar el mejoramiento de la calidad de vida. La UNA, igualmente, plantea una serie de valores institucionales que sustentan su quehacer de manera general y permite identificar su razón de ser como centro de estudios de educación superior e institución pública (UNA, 2015):

\section{ARTÍCULO 2. VALORES}

Los valores que sustentan el quehacer universitario son:

b. Compromiso social. Es la orientación de las tareas institucionales hacia el bien común, en particular hacia la promoción y consecución de una mejor calidad de vida para los sectores sociales menos favorecidos.

d. Equidad. Todos los miembros de la comunidad universitaria tienen los mismos derechos y oportunidades, sin ningún tipo de discriminación.

e. Respeto. Como garantía de la sana convivencia, se reconoce a cada miembro de la comunidad universitaria su dignidad como persona. (p. 20) 
Los valores establecidos por la UNA que permiten respaldar la acción andragógica son: el compromiso social enfocado hacia el bien común de sectores sociales menos favorecidos, la equidad entendida como la posibilidad de atender las diferencias individuales y el respeto como garantía para la sana convivencia, entendida como la interacción de una comunidad universitaria diversa.

Asimismo, la UNA plantea una serie de fines institucionales, los cuales la retan a garantizar una acción sustantiva que guarde una coherencia exquisita entre las palabras y las acciones, pues la envergadura de dichos fines es un verdadero compromiso de calidad educativa, según se puede observar en la selección de algunos de ellos (UNA, 2015):

\section{ARTÍCULO 3. FINES}

Los fines que sustentan el quehacer universitario son:

a. Diálogo de saberes. El conocimiento procedente de culturas y prácticas históricas seculares contribuye, junto con las fuentes y los procesos propios de creación de conocimiento, al desarrollo del quehacer académico universitario.

e. Identidad y compromiso. Es la identificación con los principios, valores y fines que la Universidad se ha definido y que generan un sentido de comunidad.

f. Formación integral. La Universidad se compromete en la formación de los pensadores, científicos, artistas, y en general los profesionales que, con visión humanista, la sociedad costarricense requiere para su desarrollo integral, el logro del bien común y el buen vivir.

g. Pensamiento crítico. La Universidad promueve el análisis sistemático y permanente de la realidad nacional e internacional, con el fin de determinar sus tendencias, y a partir de este conocimiento detectar sus problemas, necesidades y fortalezas, para ofrecer alternativas de solución. (p. 21)

Tal y como se colige de lo anterior, la Universidad Nacional tiene, entonces, la responsabilidad de enfrentar el desafío de garantizar el desarrollo de dichos fines, promover la innovación y la calidad académica. También, este centro de estudios es responsable de garantizar la atención oportuna de la mayor cantidad de poblaciones posibles, a través de la definición de un modelo en el que se establezcan los alcances de la acción 
didáctica, dentro del aula o fuera de ella, a la interacción comunicacional de aquellos protagonistas involucrados, determinada tanto por sus valores como por sus percepciones de vida, con una formación integral promotora del logro por el bien común así como del buen vivir.

Asimismo, la institución, a través del Consejo Universitario, en sesión ordinaria celebrada el 1 de junio del 2006, acta 2762, aprobó el Reglamento General sobre la Evaluación de los Procesos de Enseñanza y Aprendizaje de la Universidad Nacional, publicado en la Gaceta n. ${ }^{\circ}$ 11, del 23 de junio de 2006. Sus páginas iniciales determinan que el eje del proceso de enseñanza y aprendizaje es la comunidad docente y estudiantil; dentro de una relación dialógica, se implementa la construcción del conocimiento y el desenvolvimiento integral, se promueve el desarrollo de habilidades, destrezas, actitudes y valores para la realización plena del ser humano.

Además, sobre el proceso educativo se plantea, que:

Es un proceso que estimula el gusto por aprender y que incentiva el aprendizaje permanente, el aprender a aprender y la educación continua. También considera el contexto y los avances del conocimiento; incorpora las nuevas tecnologías, las que influyen significativamente en las formas de aprendizaje y el trabajo pedagógico, y las relaciones de los estudiantes con el entorno familiar, comunitario y con la sociedad en general. (UNA, 2006, p. 3)

Se observa un discurso singularizado desde una visión en la cual se valora lo pedagógico como única vía de intervención académica, a pesar de proyectar un posicionamiento crítico en la función "del estudiante" como actor principal y responsable de la apropiación del conocimiento:

En la construcción y reconstrucción de los aprendizajes el aporte de los estudiantes juega un papel decisivo. Con base en sus propios acervos de información, creencias, valores y formas culturales, son responsables activos de su propio aprendizaje y consolidan nuevos esquemas para desarrollar plenamente sus potencialidades. Los procesos de enseñanza y aprendizaje promueven en cada estudiante la apropiación del conocimiento, así como su aplicación a la resolución de problemas y generación de capacidades y actitudes asociadas a la innovación, la cooperación y el liderazgo. (UNA, 2006, p. 4.) 
Finalmente, en cuanto a los procesos de evaluación en el nivel institucional, el documento precitado la define como:

La evaluación educativa ha evolucionado y se ha enriquecido hacia una evaluación integral y continua que toma en cuenta todos los aspectos relacionados con el proceso educativo, no solo los que pueden ser verificados en un examen o en una demostración, sino todos los aspectos relacionados con el clima de trabajo en la clase y la disposición que muestren docentes y alumnos durante el desarrollo de los cursos, como puntualidad, participación, disposición al trabajo en equipo y esfuerzo personal, entre otros. (UNA, 2006, p. 4)

En este sentido, la evaluación es un proceso dinámico que, además de los logros cognoscitivos, considera el desarrollo y la modificación de habilidades, destrezas, valores y actitudes. Asimismo, es importante valorar e identificar la correspondencia con los principios fundamentales del aprendizaje de adultos, definidos por Knowles, Holton y Swanson (2005):

1. La necesidad de aprender del aprendiz (por qué, qué y cómo)

2. El concepto del alumno (autónomo y autodirigido)

3. Experiencias previas del alumno (recursos y modelos mentales)

4. Disposición para aprender (existencial y tarea de desarrollo)

5. Inclinación al aprendizaje (centrada en un problema y contextual)

6. Motivación para aprender (valor intrínseco y retribución personal). (p. 205)

Por ello, la evaluación es una oportunidad de reflexionar sobre la práctica pedagógica, permite valorar, reforzar y mejorar los procesos de enseñanza y aprendizaje, en concordancia con la naturaleza de la disciplina y la carrera, de la metodología de trabajo utilizada, los objetivos del curso y las características socioculturales del estudiantado.

Queda definido claramente el enfoque de evaluación en el nivel institucional; sin embargo, en el de aula, o bien en el nivel de microplanificación educativa, es necesario definir y ejecutar una acción docente que garantice un diseño y una praxis curricular congruente con esos principios institucionales. Lo anterior, para el desarrollo de un modelo que 
atienda los requerimientos y necesidades desde la andragogía, pues la vinculación del colectivo de estudiantes y profesores no se da de manera vertical ni unilateral, sino integrada, como se observa en la figura 2:

\section{Figura 2.}

\section{Diferencias entre pedagogía y andragogía}

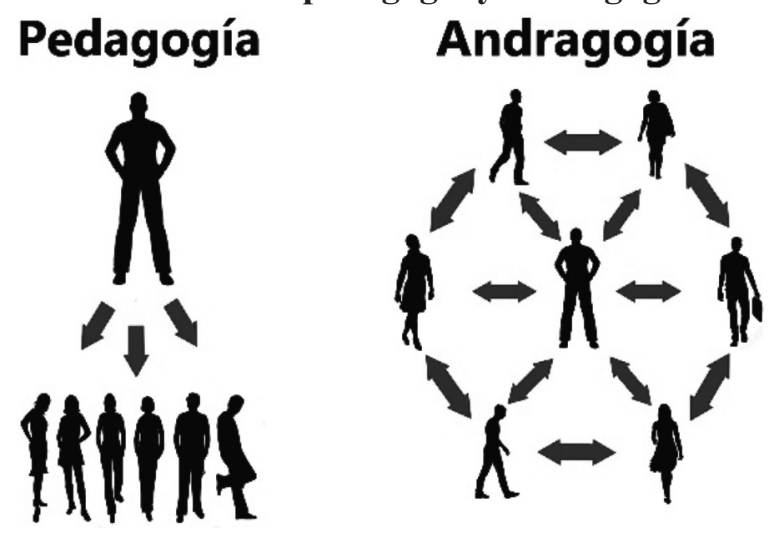

Nota: Figura de elaboración propia del autor.

En la figura 2, es importante observar, de modo gráfico, el posicionamiento de los roles de las diferentes personas involucradas en el acto educativo. Aquí se visualiza la interacción, en el caso de la pedagogía, unidimensionalmente, donde el docente es quien posee y determina la información que se brinda en el proceso; o bien, multidimensionalmente, en el caso de la andragogía, donde se valora el bagaje de conocimientos adquiridos a lo largo de la vida del colectivo estudiantil, como insumo para la mediación docente en una relación de igualdad, equidad y equilibrio de todos los actores educativos. En fin, el acto educativo cambia su mapa tradicional de verticalidad y rigidez por uno de relaciones múltiples y horizontales; ello no significa que se elimina la figura central del docente, sino que su rol cambia de una posición dominante, como responsable único y absoluto, a una de facilitador y guía, mediante la que comparte las responsabilidades con sus estudiantes.

No se puede hacer omisiva la influencia de la perspectiva de la acción individual del personal académico en el día tras día de esa acción sustantiva, donde se implementa tanto el currículum explícito, propio de los planes de estudio de cada carrera o de la educación continua, 
como el currículum, oculto conformado por todos los símbolos, significaciones, posicionamientos, actitudes y conductas presentes en las acciones individuales, colectivas y en las situaciones de aprendizaje (de forma latente o encubierta). Esto se convierte en un importante espacio de aprendizaje para cada estudiante.

Se considera importante generar diálogos para valorar, desde lo curricular, el abordaje de una población que hasta hoy se invisibiliza sistemáticamente en la formalidad del diseño para ejecutar y evaluar procesos de la educación superior en nuestro país. Asimismo, se debe reconocer que existe la plataforma necesaria, en la Universidad Nacional, para desarrollar acciones de integración y atención a aquellos estudiantes regulares pertenecientes a la población adulta, quienes también son estudiantes UNA y, tal como se señaló, cuentan con características distintivas que meritoriamente deben ser abordadas en el nivel institucional.

Por lo anterior, se debe asumir la andragogía como el espacio ideal para generar relaciones entre iguales, donde la información fluye de unos a otros, de manera dinámica y equilibrada. Además, se asume, de modo práctico, más allá de un posicionamiento discursivo, para ser implementado como un modelo sistemático que se fundamenta en tres niveles observados en la figura 3, basada en Knowles, Holton y Swanson (2005), a saber:

\section{Figura 3.}

\section{Modelo sistemático fundamentado en tres niveles}

\section{METAS Y PROPOSITOS DEL APRENDIZAJE}

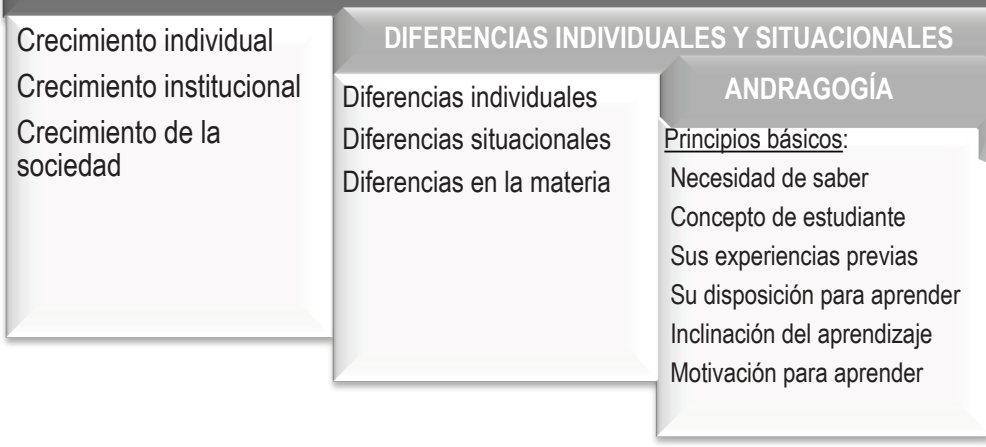

Nota: Figura basada en información de Knowles, Holton y Swanson (2005). 
En la figura 3, se visualiza claramente la importancia que tiene, para el crecimiento institucional, la valoración de la andragogía como proceso de formación de las poblaciones adultas presentes en toda universidad. En el caso del modelo sistemático integrado por tres niveles, primero se encuentran las metas y los propósitos de aprendizaje, que integran el crecimiento individual, el institucional y el de la sociedad; segundo, las diferencias individuales y situacionales, que las constituyen justamente las distinciones individuales, situacionales y aquellas en la materia; tercero, la andragogía, definida por seis principios básicos del aprendizaje de adultos, a saber: la necesidad de saber (porqué, qué y cómo), el concepto de estudiante (autónomo y autodirigido), sus experiencias previas (recursos y modelos mentales), su disposición para aprender (existencial y tarea del desarrollo), inclinación del aprendizaje (centrada en un problema y contextual) y la motivación para aprender (valor intrínseco y retribución personal).

Por lo tanto, hacer realidad la atención de la población adulta dentro de la UNA hace necesaria la definición de acciones enmarcadas en un modelo pedagógico y andragógico, integrador de las realidades institucionales. En estas últimas, es imperativa la promoción de los espacios de sensibilización y capacitación a toda la comunidad universitaria en dicho tema como rasgo distintivo de un enfoque humanista, además de ser esencial instar la igualdad de oportunidades, así como la garantía de la atención integral y la participación de toda la comunidad universitaria.

De esta forma, se requiere generar los espacios, para pasar de las palabras a los hechos, en cuanto a promover una institución respetuosa e integradora que permita las condiciones adecuadas para la permanencia de nuestra comunidad estudiantil en los diversos programas de formación que desarrolla la UNA.

\section{Referencias}

Alonso Chacón, P. (2012). La andragogía como disciplina propulsora de conocimiento en la educación superior. Revista Electrónica Educare, 16(1), 15-26. Recuperado de http://www.revistas.una. ac.cr/index.php/EDUCARE/article/download/3729/3578

Knowles, M., Holton, E. y Swanson, R. (2005). Andragogía: El aprendizaje de los adultos. México: Alfaomega. 
Lloyd, R. (2015). Pedagogía vs. Andragogía. Recuperado de http:// obrerofiel.com/pedagogia-vs-andragogia/

Pérez, S. (2009). Modelo Andragógico. Fundamentos. Universidad Valle de México. México D. F.: Ma. Guadalupe Ambriz. Recuperado de: https://my.laureate.net/faculty/docs/Faculty\%20Documents/Andragogia.Fundamentos.pdf

Universidad Nacional. (2006). Reglamento general sobre los procesos de enseñanza y de aprendizaje de la Universidad Nacional. Heredia: Programa de Publicaciones de la Universidad Nacional.

Universidad Nacional. (2015). Estatuto Orgánico. Recuperado de http://www.documentos.una.ac.cr/bitstream/handle/unadocs/6693/ESTATUTO-ORG\%C3\%81NICO-UNA-digital. pdf? sequence $=1 \&$ isAllowed $=\mathrm{y}$

Universidad Nacional. (2015). Modelo Pedagógico. Recuperado de http:// www.documentos.una.ac.cr/bitstream/handle/unadocs/1763/modelo_pedagogico_UNA.141.pdf? sequence $=3$

Universidad Nacional. (2015). Reglamento de Evaluación. Recuperado de http://www.defensoria.una.ac.cr/documentos/normas_generales.pdf 